\title{
5. Jones-matrix mapping of complex degree of mutual anisotropy of birefringent protein networks during the differentiation of myocardium necrotic changes
}

\author{
Yu.A. Ushenko, V.T. Bachinskyi, O.Ya. Vanchuliak, \\ O.V. Dubolazov, M.S. Garazdyuk, V.A. Ushenko
}

\subsection{Introduction}

Optical methods of diagnostics of biological tissues include three directions:

- Spectral methods [1-3] based on investigation of absorption spectra of biological objects and media.

- Polarization methods [4-7]. The main disadvantage of such a mehtods consists in the azimuthal dependence of the obtained data on the rotation angle of the sample in relation to the probing beam [8-12]. Therefore the results of polarization mapping are hardly reproducible.

- Correlation methods are devoid of this disadvantage [13-15]. In [16] a new azimuthally stable parameter - complex degree of mutual polarization (CDMP) $W\left(d_{1}, d_{2}, t\right)$ is introduced for characterizing the correlation between the polarization states the object field in the points $\left(d_{1}, d_{2}\right)$ with the intensities $I\left(d_{1}, t\right), I\left(d_{2}, t\right)$

$$
W\left(d_{1}, d_{2}\right)=\frac{\left(E_{x}\left(d_{1}\right) E^{*}{ }_{x}\left(d_{2}\right)+E_{y}\left(d_{1}\right) E^{*}{ }_{y}\left(d_{2}\right)\right)^{2}}{I\left(d_{1}\right) I\left(d_{2}\right)} .
$$

For the purpose of practical applications in the field of investigation of optically anisotropic tissues another form of (5.1) is used [17-20] 


$$
\operatorname{Re}\{W\} \equiv \tilde{W}\left(d_{1}, d_{2}\right)=\frac{\left\{E_{x}\left(d_{1}\right) E_{x}\left(d_{2}\right)-E_{y}\left(d_{1}\right) E_{y}\left(d_{2}\right) \cos \left[\theta\left(d_{1}\right)-\theta\left(d_{2}\right)\right]\right\}^{2}}{I\left(d_{1}\right) I\left(d_{2}\right)},
$$

were $\theta\left(d_{1}\right)$ и $\theta\left(d_{2}\right)$ - the phase shifts between the orthogonal components $E_{x}, E_{y}$ of amplitude of laser beam.

In [17-20] relation (5.2) takes into consideration linear birefringence of biological tissues fibrillar networks. However, taking into account the circular birefringence of protein molecules, which form the fibrillar networks, is topical. In [21] the technique of differentiation of such mechanisms of anisotropy using of direct and inverse Fourier transform of microscopic images of these objects is proposed.

This research presents the possibilities of diagnostics of myocardium necrotic changes. At present time postmortem diagnostics of acute coronary syndrome is a leading approach in morphological $[22,23]$ and forensic [24, 25] investigations. Balanced accuracy [26-28] of acute coronary insufficiency detection by conventional histochemical methods does not exceed 65\%-70\% and takes relatively long time. Therefore, topical is the development of new, more accurate and express methods utilizing of novel optical-physical approaches to formation and processing of microscopic images of myocardium histological sections.

This work is aimed at the development and substantiation of the method Jones-matrix mapping and spatial-frequency filtering of manifestations of optical anisotropy caused by the necrotic changes of histological sections of myocardium tissue of those who have died of ischemic heart disease (IHD) and acute coronary insufficiency (ACI).

Two types of optical anisotropy simultaneously exist in biological tissues [11]. There are: 
- phase anisotropy (linear and circular birefringence);

- amplitude anisotropy (linear and circular dichroism).

Optical manifestations of such a mechanisms connected with morphological structure of biological tissues. So, circular birefringence and dichroism connected with spiral-like structure of protein molecules. Linear birefringence and dichroism appear as the result of formation by such structures of spatially ordered fibrillar networks.

Mechanisms of phase and amplitude anisotropies of protein structures can be separated by choosing of spectral region. Absorption of proteins is minimal at long-wave region of spectrum. Due to this fact in our spectral region of radiation of He-Ne laser $(\lambda=0.6328 \mu \mathrm{m})$ optical anisotropy of myocardium tissue consists of the combination of circularly birefringent molecules of myosin, which form linear birefringence of fibers and bundles of fibrillar network.

Let's consider detailed analytical description of the processes of transformation of laser radiation by myocardium tissue [6-12]:

- there are two scales of spatial organization of optically anisotropic components of myocardium tissue;

- components $(l \sim 5 \mu m \div 15 \mu m)$ of optically anisotropic molecules of myosin with prevailing circular birefringence form small scale;

- components $(l \sim 50 \mu m \div 200 \mu m)$ of fibrillar optically anisotropic network of myosin with prevailing (due to spatial ordering of the directions of optical axes of partial biological crystals) linear birefringence form large scale;

- polarization properties of the points of such optically anisotropic layer are characterized by the following matrix of optical anisotropy $\{F\}$ 


$$
\{F\}=\{L\}\{C\}
$$

where $\{L\}$ - Jones matrix of linear birefringence; $\{C\}$ - Jones matrix of circular birefringence or optical activity [20]

$$
\left\{\begin{array}{l}
f_{11}=\left[\sin ^{2} \rho+\cos ^{2} \rho \exp (-i \omega)\right] \cos \psi+[\sin \rho \cos \rho(1-\exp (-i \omega))] \sin \psi ; \\
f_{12}=-\left[\sin ^{2} \rho+\cos ^{2} \rho \exp (-i \omega)\right] \sin \psi+[\sin \rho \cos \rho(1-\exp (-i \omega))] \cos \psi ; \\
f_{21}=[\sin \rho \cos \rho(1-\exp (-i \omega))] \cos \psi+\left[\cos ^{2} \rho+\sin ^{2} \rho \exp (-i \omega)\right] \sin \psi \\
f_{22}=-[\sin \rho \cos \rho(1-\exp (-i \omega))] \sin \psi+\left[\cos ^{2} \rho+\sin ^{2} \rho \exp (-i \omega)\right] \cos \psi
\end{array}\right.
$$

here $\rho$ - optical axis direction, $\omega=(2 \pi / \lambda) \Delta n p-$ phase shift value between the orthogonal components of the laser wave amplitude with wavelength $\lambda$, which passed the geometrical path $p$ through the biological crystal with linear birefringence $\Delta n ; \omega$ - angle of rotation of polarization plane of laser beam caused by circular birefringence.

For azimuthally stable situation of biological tissue probing by circularly polarized beam, taking into account (5.4), we obtain the interconnection between the optical anisotropy of the layer and the polarization structure of its image.

$$
\left.\left(\begin{array}{l}
E_{x}(d) \\
E_{y}(r)
\end{array}\right)=\frac{1}{\sqrt{2}} \| \begin{array}{ll}
f_{11}(d) & f_{12}(d) \\
f_{21}(d) & f_{22}(d)
\end{array}\right)\left(\begin{array}{l}
1 \\
i
\end{array}\right)=\frac{1}{\sqrt{2}}\left(\begin{array}{l}
f_{11}(d)+i f_{12}(d) \\
f_{21}(d)+i f_{22}(d)
\end{array}\right) .
$$

Relation (5.5) can be rewritten as a "one-point" interconnection of the optically anisotropic parameters and amplitude $\left\{\begin{array}{l}E_{x}(d)=g(\rho, \omega, \psi) ; \\ U_{y}(d)=j(\rho, \omega, \psi) .\end{array}\right.$

Due to "two-point" approach [16-19] similarly to the CDMP parameter $W\left(d_{1}, d_{2}\right)(5.1)$ parameter of Jones-matrix correlation $H\left(d_{1}, d_{2}\right)-$ 
the complex degree of mutual anisotropy (CDMA) - can be introduced. It directly characterizes the degree of consistency of parameters of linear and circular birefringence in the points of the biological tissue $W\left(\begin{array}{l}E_{x}\left(d_{1}, d_{2}\right) \\ E_{y}\left(d_{1}, d_{2}\right)\end{array}\right) \rightarrow H\left(\begin{array}{l}g\left(f_{i k}\left(d_{1}, d_{2}\right)\right) \\ j\left(f_{i k}\left(d_{1}, d_{2}\right)\right)\end{array}\right)$. In order to determine the analytical expression for CDMA we have used the relation (5.1) taking into account the relations $(5.3)-(5.5)$

$$
H\left(d_{1}, d_{2}\right)=\frac{\left\{f_{11}\left(d_{1}\right) f_{11}\left(d_{2}\right)+f_{12}\left(d_{1}\right) f_{12}\left(d_{2}\right)+f_{21}\left(d_{1}\right) f_{21}\left(d_{2}\right)+f_{22}\left(d_{1}\right) f_{22}\left(d_{2}\right)\right\}^{2}}{I\left(d_{1}\right) I\left(d_{2}\right)},
$$

As the result we have obtained the new correlation parameter $H\left(d_{1}, d_{2}\right)$ describing generalized anisotropy of biological tissues layers. Let's consider the spatial-frequency filtering for separation of coordinate distributions of CDMA formed by various components of the diagnosed layer of myocardium tissue with linear and circular birefringence.

The task of optical selection of polarization appearances of the linear $(\rho, \omega)$ and circular $(\psi)$ birefringence of the network of myosin fibrils of myocardium tissue for the differentiation of the cause of death from the viewpoint of medicine is topical. It follows from fact that in the case of death due to ACI - there are necrotic changes of myosin structures on small-scale range. From the optical point of view such process reveals in degradation of circular birefringence $(\psi \downarrow)$ of the fibrillar network. Death due to IHD accompanied by orientation changes of large-scale fibrillar network with the degradation of linear birefringence $(\omega \downarrow)$. Traditional histological diagnostics and differentiation of such necrotic changes is rather labor-intensive, it requires more time and often appears to be ambiguous. Let's return to the analysis of interconnections ((5.1)-(5.6)) 
between the biological layer image polarization structure and parameters describing it anisotropy. According to the proposed model of such anisotropy the linear birefringence is typical mainly for large-scale fibrillar networks of myosin fibrils. In the case of small-scale structures the circular birefringence is typical. Thus spatially high-frequency $(E(\rho, \omega))$ and lowfrequency $(E(\psi))$ components are formed in the image of such an object. For the purpose of a selection of above mentioned conditions the method of spatial-frequency filtration of the polarization inhomogeneous laser images in Fourier plane has been applied [21].

The main idea of such approach is that spatial-frequency structure of the Fourier spectrum of myocardium tissue laser image is different for its large-scale and small-scale protein structures. Therefore, usage of spatialfrequency filtration brings the possibility for isolation either of lowfrequency (with linear birefringence) or of high-frequency (with circular birefringence) components, which by means of Fourier transform can be transformed into respectively "separated" laser images. By mean of location of vignetting (transparent $L(\Delta \chi, \Delta \gamma)$ or opaque $L^{-1}(\Delta \chi, \Delta \gamma)$ ) diaphragm in the central part of Fourier plane using of reverse Fourier transform it is possible to restore the low- and high-frequency components $\left\{\begin{array}{l}\hat{E}(\rho, \omega, \chi, \gamma)=L(\Delta \chi, \Delta \gamma) E(\chi, \gamma) ; \\ \hat{E}(\psi, \chi, \gamma)=L^{-1}(\Delta \chi, \Delta \gamma) E(\chi, \gamma) .\end{array} \quad\right.$ Here $\quad \chi=\frac{x}{\lambda f} \quad$ and $\gamma=\frac{y}{\lambda f} \quad-\quad$ spatial frequencies.

Therefore, coordinate distributions of the Jones matrix elements of linear $q_{i k}(\rho, \omega)=F(\hat{U})$ and circular $a_{i k}(\psi)=F\left(U^{\&}\right)$ birefringence can be measured according to classical technique [20]. As the result we obtain the analytical expressions of the CDMA parameter $\left(H\left(\rho, \omega, d_{1}, d_{2}\right), H\left(\psi, d_{1}, d_{2}\right)\right)$ for various types of optically anisotropic layers of myocardium tissue. 
Experimental confirmation of the efficiency of spatial-frequency separation of optical manifestations of phase anisotropy mechanisms of different scale structures of myocardium proved in the symmetry of Jones matrix. Thus, for the linear birefringence - all matrix elements $f_{i k} \neq 0$ (object field is formed by elliptically polarized fragments); for circular birefringence $f_{12 ; 21} \rightarrow 0 ; f_{11} \approx f_{22}$ (object field is formed by linearly polarized fragments).

\subsection{Spatial-Frequency Filtering of CDMA Coordinate Distributions}

Experimental investigations of CDMA coordinate distributions were performed in the classical setup of polarimeter [5]. Modified setup of such laser Stokes-polarimeter with spatial-frequency filtration is presented in Fig. 5.1.

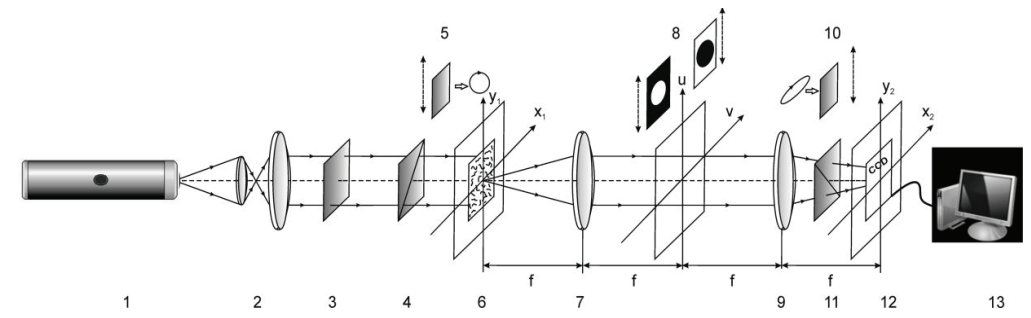




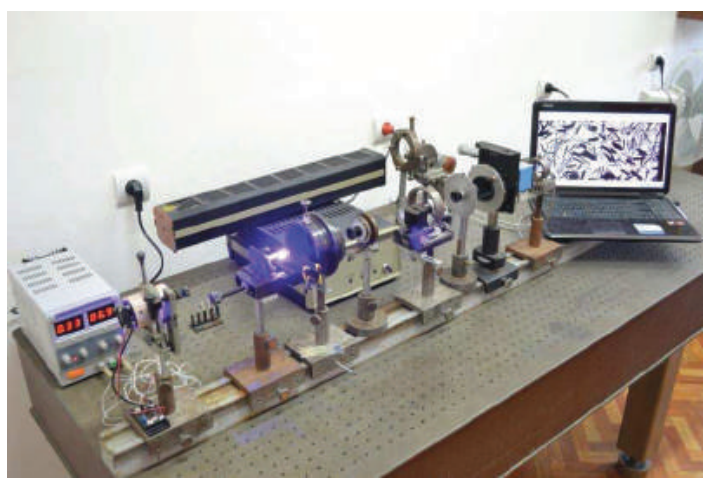

Fig. 5.1. Experimental setup of Stokes-polarimeter with spatial-frequency filtering. Here 1 - He-Ne laser; 2 - collimator; 3 - stationary quarterwave plate; 5, 10 - mechanically movable quarterwave plates; 4, 11 - polarizer and analyzer respectively; 6 - object of investigation; 7, 9 - strain-free objectives; 8 - low-frequency and high-frequency filters, 12 - CCD camera; 13 - personal computer

Illumination of the investigated sample 6 was performed by parallel $\left(\varnothing=10^{4} \mu \mathrm{m}\right) \mathrm{He}-\mathrm{Ne}(\lambda=0.6328 \mu \mathrm{m}$; power $W=5 \mathrm{~mW})$ laser beam 1,2 . The polarization light source consists of quarter-wave plates 3,5 (Achromatic True Zero-Order Waveplate) and polarizer 4; this enables to form a laser beam with random azimuth $0^{\circ} \leq \alpha_{0} \leq 180^{\circ}$ or ellipticity $0^{0} \leq \beta_{0} \leq 90^{\circ}$ of polarization. Myocardium histological sections 6 were located in the focal plane of strain-free objective 7 (Nikon CFI Achromat P, working distance $-30 \mathrm{~mm}, N A-0.1$, magnification $-4 \mathrm{x}$ ). In the back focal plane a spatial-frequency (a low-frequency $L(\Delta \chi, \Delta \gamma)$ or high-frequency $\left.L^{-1}(\Delta \chi, \Delta \gamma)\right)$ filter 8 was located. Strain-free objective 9 was located at the focal distance from frequency plane of objective 7 and, therefore, 
performed an inverse Fourier-transform of spatially filtered field of laser radiation. The coordinate distribution of intensity of such field was registered in the plane of light-sensitive CCD-camera 12 (The Imaging Source DMK 41AU02.AS, monochrome 1/2" CCD, Sony ICX205AL (progressive scan); resolution - 1280x960; size of light-sensitive plate 7600x6200 $\mu \mathrm{m}$; sensitivity - 0.05 lx; dynamic range -8 bit; SNR - 9 bit, deviation of photosensitive characteristics from the linear one does not exceed 5\%) that was also located at the focal distance from objective 9 and provided the measurement range of the structural elements of the restored image of myocardium tissue histological sections for geometrical sizes 2$2000 \mu \mathrm{m}$.

\subsection{Results and Discussion}

The experimental measurement of CDMA components - complex elements $d_{i k}$ of Jones matrix - is based on the approach suggested in [20].

1. Determination of statistically reliable representative selection of patients with the known (referent) diagnosis

As objects of investigation we chose optically thin (attenuation coefficient $\tau \approx 0.091 \div 0.097$ ) histological sections of biopsy of myocardium tissue. In this case in the volume of such layers single scattering regime is realized. In other words there is no depolarization (multiple scattering), but only the transformation of polarization, which is accompanied by the formation of polarization-inhomogeneous images of myocardium samples.

From the medical point of view two groups of patient with the following diagnoses, prepared according to standard technique on the freezing microtome were formed:

-group 1 - dead due to IHD; 
- group 2 - dead due to ACI.

In our case group 1 plays the role of control and group 2 - diagnosed.

The differentiation of IHD (group 1) and ACI (group 2) was determined by the gold standard method - biopsy of surgically removed myocardium. A reliable number of people $(n=51)$ was determined by means of software product Statmate for $95 \%$ confidence interval $(p<0.05)$. The value of representative sampling was verified by the cross-validation method. Root-mean-square deviation of the average value of statistical moments $M_{i=1 ; 2 ; 3 ; 4}$ does not exceed 0.025; this corresponds to the value of statistically reliable confidence interval $p<0.05$.

\section{Samples preparation}

Optically thin histological sections of myocardium tissue biopsy were prepared on freezing microtome according to standard technique.

\section{Algorithm of the analysis of CDMA coordinate distributions}

The measured distributions $q \equiv\left\{H_{\rho, \omega}, H_{\psi}\right\}$ were objectively analyzed within the statistical approach - the set of statistical moments of the $1^{\text {st }}-4^{\text {th }}$ order was determined

$$
\begin{aligned}
& M_{1}=\frac{1}{S} \sum_{j=1}^{S} q_{j} ; M_{2}=\sqrt{\frac{1}{S} \sum_{j=1}^{S}\left(q-M_{1}\right)_{j}{ }^{2}} ; M_{3}=\frac{1}{M_{2}{ }^{3}} \frac{1}{S} \sum_{j=1}^{S}\left(q-M_{1}\right)_{j}^{3} ; \\
& M_{4}=\frac{1}{M_{2}{ }^{4}} \frac{1}{S} \sum_{j=1}^{S}\left(q-M_{1}\right)_{j}{ }^{4} .
\end{aligned}
$$


Here $S$ - number of pixels of CCD-camera. These parameters characterize the mean $\left(M_{1}\right)$, dispersion $\left(M_{2}\right)$, skewness $\left(M_{3}\right)$ and kurtosis or "peak sharpness" $\left(M_{4}\right)$ of histograms $N(q)$.

4. Jones-matrix mapping of CDMA coordinate distributions of myocardium histological sections with spatial-frequency selection.

We have used a classical approach proposed in monography [20] for experimental measurements of Jones matrix elements within each pixel of $\mathrm{CCD}$ camera. Jones matrix can be written as the combination of real and imaginary (phase angles) components. The values of real components of Jones matrix elements can be measured as follows:

- sample was illuminated by linearly polarized beam with azimuth $0^{0}$;

- transmission plane of analyzer was rotated by angles of $0^{\circ} ; 90^{\circ}$ and the corresponding intensities of transmitted radiation were measured;

- sample was illuminated by linearly polarized beam with azimuth $90^{\circ}$;

- transmission plane of analyzer was rotated by angles of $0^{\circ} ; 90^{\circ}$ and the corresponding intensities of transmitted radiation were measured;

- real components of Jones matrix elements were calculated.

The values of imaginary components of Jones matrix elements can be measured as follows:

- sample was illuminated by right circularly polarized beam (angle between the transmission plane of polarizer and fast axis of quarterwave plate was $45^{\circ}$ );

- transmission plane of analyzer was rotated by angles of $0^{0} ; 90^{\circ}$ and the corresponding intensities of transmitted radiation were measured;

- sample was illuminated by linearly polarized beam with azimuth $45^{0}$; 
- transmission plane of analyzer was rotated by angles of $0^{0} ; 90^{\circ}$ and the corresponding intensities of transmitted radiation were measured;

- imaginary components of Jones matrix elements were calculated.

High- and low-frequency filtering of polarization-inhomogeneous object field of myocardium histological sections was performed by using of transparent and opaque diaphragms (Fig. 5.1, fragment 8).

For the purpose of choosing of diaphragm size the dependencies of values of all statistical moments from the size of spatial-frequency diaphragm were investigated. It was determined that beginning from the specific size of spatial-frequency filter ( $\Delta R=125$ pixels) the change of values of the set of statistical moments reached its saturation. As the criterion of optimal size we chose the condition of simultaneous extremal values of all statistical moments. For low-frequency filter (transparent) the diaphragm size was $\Delta \mathrm{R}=30$ pixels - transmission of spatial frequencies up to $v=10$ $\mathrm{mm}^{-1}$. Optimal size of high-frequency filter (opaque) was $\Delta R^{-1}=60$ pixels transmission of spatial frequencies from $v=20 \mathrm{~mm}^{-1}$.

Efficiency of spatial-frequency filtering of manifestations of linear and circular birefringence was estimated in accordance with the symmetry of Jones matrixes and prevailing polarization type high- and low-frequency components of images of myocardium samples.

It was determined that in the case of low-frequency filtering all elements of Jones matrix $f_{i k} \neq 0$. In addition, $94 \%$ of the pixels of digital camera recorded elliptically polarized areas of the myocardium image. In the case of high-frequency filtering the following results were obtained $f_{12 ; 21} \rightarrow 0 ; f_{11} \approx f_{22}$ ( $91 \%$ of linearly polarized areas).

The values of CDMA were calculated as follows: 
- under different diaphragming conditions the two-dimensional arrays of values of Jones matrix elements were measured within each pixel;

- for each line in two-dimensional array of Jones matrix elements a pairs of neighbor pixels were chosen;

- for each pair of such pixels one value of CDMA was calculated;

- at the next stage by means of scanning with the step of one pixel a set of CDMA values was determined within the chosen line of CCD camera;

- above mentioned scanning was performed within all lines of light sensitive area of CCD camera and the two-dimensional array of CDMA values was calculated.

The figures 5.2 and 5.3 present the results of Jones-matrix mapping of low-frequency (linear birefringence of fibrillar networks - Fig. 5.2) and high-frequency (circular birefringence of myosin molecules - Fig. 5.3) CDMA distributions of myocardium tissue histological sections. Figures consists of the coordinate distributions (fragments (1),(3)) and histograms $N(q)$ (fragments (2),(4)) of sample randomly taken from group 1 (fragments (1),(2)) and from group 2 (fragments (3),(4)).

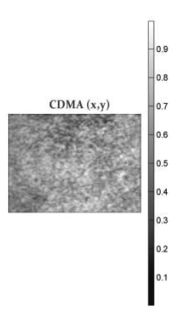

1)

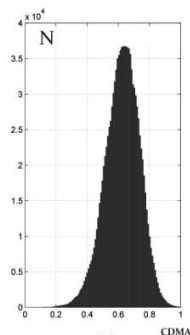

2)

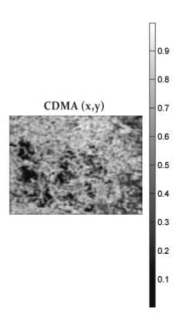

3)

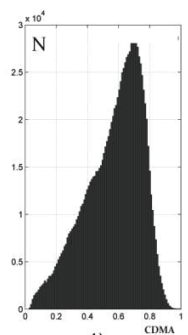

4)

Fig. 5.2. Coordinate distribution $((1),(3))$ and histograms ((2),(4)) of CDMA of linear birefringence of histological sections of myocardium tissue of group $1((1),(2))$ and group $2((3),(4))$. 


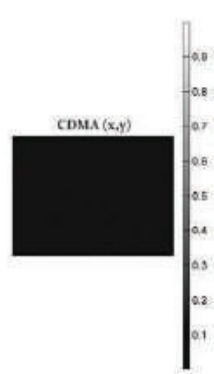

1)

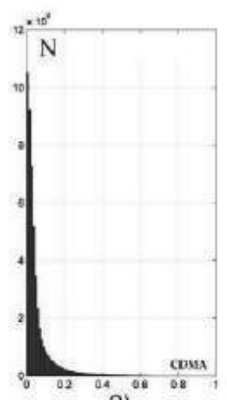

2)

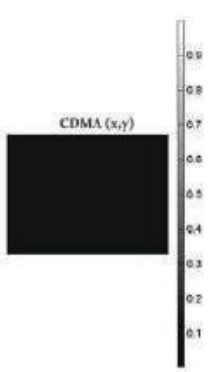

3)

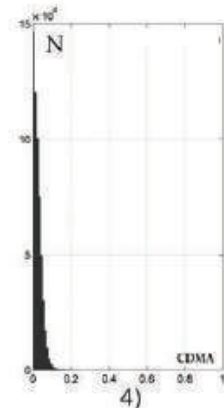

Fig. 5.3. Coordinate distribution $((1),(3))$ and histograms $((2),(4))$ of CDMA of circular birefringence of histological sections of myocardium tissue of group $1((1),(2))$ and group 2 ((3),(4)).

CDMA $H_{\rho, \omega}$. The comparative analysis of the spatial-frequency filtered coordinate distributions $H_{\rho, \omega}(m \times n)$ of large-scale network of myocardium layer revealed certain difference between them. The histograms of the CDMA distribution for a histological section of myocardium from group 2 (ACI) are characterized by asymmetric structure (Fig. 5.2, fragment (4)). The similar distribution found for the tissue sample from group 1 (IHD) (Fig. 5.2, fragment (2)) is more symmetrical. The revealed peculiarity is related to the structure of myosin fibrils. For histological sections of group 2 the directions of the myosin fibrils are more ordered in the direction of optical axes. For the samples from group 1 the similar network of myocardium layer is more disordered in directions. From the optical point of view this geometrical structure $\left(\rho^{*} \leftrightarrow N_{\max }\right)$ reveals in the formation of the priority, most probable values of CDMA $H_{\rho, \omega}(m \times n)$ (relations (5.1), (5.3)-(5.6)), the set of which forms the main extreme. Statistically such a process is detected by the following changes 
of $M_{i=1 ; ; ; 3 ; 4}\left(H_{\rho, \omega}\right)$ (relations (5.7)). For the IHD - the decrease of the skewness $M_{3} \downarrow$ and the kurtosis $M_{4} \downarrow$ of histograms $N\left(H_{\rho, \omega}\right)$. Other statistical moments (mean $M_{1} \uparrow$, dispersion $M_{2} \uparrow$ ) increase. Thus "CDMA $H_{\rho, \omega}$ - biopsy" appeared to be sensitive to the changes of linear birefringence caused by necrotic transformation of fibrillar networks of myocardium. That is why this information is important for differentiation of causes of death due to IHD and ACI.

CDMA $H_{\psi}$. The physical mechanism of circular birefringence formation is first of all related to concentration of optically active protein molecules of myosin, which form the myocardium fibrillar networks. Necrotic changes caused by ACI lead to decrease of concentration of protein molecules. Thus the correlation parameter $W_{\theta}$ of such layers is characterized by the less magnitude of values in comparison with the CDMA distribution obtained for myocardium from group 1. Quantitatively such difference characterizes histograms $N\left(H_{\psi}\right)$ (Fig. 5.3, fragment (2)). It is obvious from the obtained data (Fig. 5.3, fragment (4)) that for necrotic changes caused by ACI less in magnitude values of the average $M_{1}\left(H_{\psi}\right) \downarrow$, dispersion $M_{2}\left(H_{\psi}\right) \downarrow$ are typical. The skewness $M_{3}\left(H_{\psi}\right) \uparrow$ and the kurtosis $M_{4}\left(H_{\psi}\right) \uparrow$ of distributions $N\left(H_{\psi}\right)$ increases. This fact indicates to sensitivity “CDMA $H_{\psi}$ - biopsy" to "concentration" changes of circular birefringence of myocardium. That is why such information is the most important in the ACI diagnostics of myocardium.

\section{Statistical analysis}

The differentiation between the group 1 and group 2 was determined by using of the following methodology [26-28]: 
- within each set of values of statistical moments $M_{i=1 ; 2 ; 3 ; 4}$ we determined the average value $\bar{M}_{i=1 ; 2 ; 3 ; 4}$ and standard deviation $\sigma_{i=1 ; 2 ; 3 ; 4}$;

- differences between the statistical sets $M_{i=1 ; 2 ; 3 ; 4}$ were significant in the case when the average value $\bar{M}_{i=1 ; 2 ; 3 ; 4}$ within the group 1 didn't "overlap" with the standard deviation $\sigma_{i=1 ; 2 ; 3 ; 4}$ within group 2 and vice versa.

- within both groups of myocardium samples for the distributions of values of each statistical moments $M_{i=1 ; 2 ; 3 ; 4}$ we chose cutoff of $3 \sigma$ (99.72\% of all possible values of changes of $M_{i}$ ). Sequentially, we determined the number of "false negative" $(b)$ and "false positive" (d) conclusions;

- For every statistical moments traditional for probative medicine operational characteristics: sensitivity $\left(S e=\frac{a}{a+b} 100 \%\right)$, specificity $\left(S p=\frac{c}{c+d} 100 \%\right)$ and balanced accuracy $\left(A c=\frac{S e+S p}{2}\right)$, where $a$ and $b$ are the number of correct and wrong diagnoses within group 2; $c$ and $d$ - the same within group 1 were determined.

Table 5.1. Parameters of statistical structure of CDMA coordinate distributions

\begin{tabular}{|c|c|c|c|c|}
\hline \multirow{2}{*}{ Parameters } & \multicolumn{2}{|c|}{$H_{\rho, \omega}$} & \multicolumn{2}{c|}{$H_{\psi}$} \\
\cline { 2 - 5 } & IHD & ACI & IHD & ACI \\
\hline$M_{1}$ & $0.76 \pm 0.054$ & $0.72 \pm 0.051$ & $0.09 \pm 0.005$ & $0.06 \pm 0.004$ \\
\hline$M_{2}$ & $0.21 \pm 0.014$ & $0.24 \pm 0.015$ & $0.11 \pm 0.007$ & $0.08 \pm 0.006$ \\
\hline$M_{3}$ & $0.39 \pm 0.023$ & $0.21 \pm 0.016$ & $0.71 \pm 0.057$ & $1.21 \pm 0.084$ \\
\hline$M_{4}$ & $0.58 \pm 0.035$ & $0.72 \pm 0.15$ & $1.23 \pm 0.091$ & $1.92 \pm 0.14$ \\
\hline
\end{tabular}


The comparative analysis of the data obtained (Table 5.1) showed that the differences between the values of average $\bar{M}_{i=1 ; 2 ; 3 ; 4}$ moments of all orders are statistically valid. However, there is an intergroup overlap for all histograms $N\left(M_{i}\right)$. Moreover, the range of such an overlap is inversely proportional to the value of the difference between the averages $\bar{M}_{i=1 ; ; ; 3 ; 4}$. The moments $M_{i=3 ; 4}\left(H_{\rho, \omega}\right)$ appeared to be sensitive in differentiation of linear birefringence maps $H_{\rho, \omega}(m \times n)$ of myocardium histological sections (highlighted in grey in Table 1). For circular birefringence distributions $H_{\psi}(m \times n)$ of myocardium layers the most preferred are also higher-order statistical moments $M_{i=3 ; 4}\left(H_{\psi}\right)$.

Table 5.2 presents the parameters of information value of azimuthally stable method of Jones-matrix mapping of optical anisotropy of histological sections of biopsy of myocardium with different necrotic changes.

Table 5.2. Operational characteristics of the method of Jones-matrix mapping of optical anisotropy of histological sections of biopsy of myocardium

\begin{tabular}{|c|c|c|c|}
\hline Parameters & $M_{i}$ & $H_{\rho, \omega}$ & $H_{\psi}$ \\
\hline \multirow{3}{*}{$A c\left(M_{i}\right)$} & $M_{1}$ & $56 \%$ & $67 \%$ \\
\cline { 2 - 4 } & $M_{2}$ & $58 \%$ & $65 \%$ \\
\cline { 2 - 4 } & $M_{3}$ & $93 \%$ & $89 \%$ \\
\cline { 2 - 4 } & $M_{4}$ & $91 \%$ & $92 \%$ \\
\hline
\end{tabular}

The obtained results enable to state a rather high level of accuracy of azimuthally stable Jones-matrix mapping. According to the criteria of probative medicine [26] the parameters $A c(\psi) \sim 90 \%$ correspond to good quality, while $A c(\rho, \omega)>90 \%$ - to high quality.

6. Possibilities of clinical application 
The suggested method is more objective and express (the time of getting the result is $t \leq 15$ minutes). That is why it can be used as a preliminary one in histology for differentiation of causes of death due to IHD and ACI. Here the "CDMA $H_{\rho, \omega}$ - biopsy" is the most accurate and informative for IHD. In the task of early detection of ACI changes the use of "CDMA $H_{\psi}$ - biopsy" is more proper. Still it is too early to claim that the investigation of this problem is completed. Thus to implement this method into routine laboratory practice numerous clinical tests are required in forensic medicine.

\subsection{Conclusion}

The efficiency of the developed technique of spatial-frequency Fourier polarimetry of CDMA in the diagnostics of the necrotic changes of myocardium tissue was demonstrated.

The differentiation criteria between the causes of death due to IHD and ACI on the basis of the statistical (statistical moments of the $1^{\text {st }}-4^{\text {th }}$ order) analysis of the spatial-frequency filtered distributions of CDMA of myocardium tissue are defined.

\section{References}

1. V.V. Tuchin, Tissue Optics: Light Scattering Methods and Instruments for Medical Diagnosis, second edition, PM 166, SPIE Press, Bellingham, WA (2007).

2. X. Wang, G. Yao, L. - H. Wang, "Monte Carlo model and single-scattering approximation of polarized light propagation in turbid media containing glucose," Appl. Opt. 41, 792-801 (2002).

3. X. Wang, L. - H. Wang, "Propagation of polarized light in birefringent turbid media: a Monte Carlo study,” J. Biomed. Opt. 7, 279-290 (2002). 
4. O. V. Angelsky, P. V. Polyanskii, I. I. Mokhun, C. Yu. Zenkova, H. V. Bogatyryova, Ch. V. Felde, V. T. Bachinskiy, T. M. Boichuk and A. G. Ushenko, "Optical Measurements: Polarization and Coherence of Light Fields, Modern Metrology Concerns, Edited by Luigi Cocco, ” ISBN 978-953-510584-8, Hard cover, 458 pages, Publisher: InTech, Published: May 16, 2012 under CC BY 3.0 license, in subject Physical Sciences, Engineering and Technology.

5. Y. A. Ushenko, T. M. Boychuk, V. T. Bachynsky, O. P. Mincer, "Diagnostics of Structure and Physiological State of Birefringent Biological Tissues: Statistical, Correlation and Topological Approaches" in Handbook of CoherentDomain Optical Methods, Springer Science+Business Media, p. 107, New York (2013).

6. O.V. Angelsky, C. Yu. Zenkova, M.P. Gorsky, N.V. Gorodyns'ka, "Feasibility of estimating the degree of coherence of waves at the near field," Applied Optics 48, 2784-2788 (2009).

7. Yu. A. Ushenko, "Statistical structure of polarization-inhomogeneous images of biotissues with different morphological structures," Ukr. J. Phys. Opt. 6, 6370 (2005).

8. O.V. Angelsky, A.G. Ushenko, and Yu.A. Ushenko, "Polarization reconstruction of orientation structure of biological tissues birefringent architectonic nets by using their Mueller-matrix speckle-images," J. Holography Speckle 2, 72-79 (2005).

9. O.V. Angelsky, A.G. Ushenko, Y.G. Ushenko, "2-D Stokes polarimetry of biospeckle tissues images in pre-clinic diagnostics of their pre-cancer states," Journal of Holography and Speckle 2, 26-33 (2005).

10. O. V. Angelsky, A. G. Ushenko, Yu. A. Ushenko, V. P. Pishak, A. P. Peresunko, "Statistical, Correlation and Topological Approaches in Diagnostics of the Structure and Physiological State of Birefringent Biological Tissues" in 
Handbook of Photonics for Biomedical Science, pp. 283-322 ed. by Valery V. Tuchin, CRC PressTaylor\&Francis group: Boca Raton, London, New York (2010).

11. Yu. A. Ushenko, T. M. Boychuk, V. T. Bachynsky, O. P. Mincer, "Diagnostics of Structure and Physiological State of Birefringent Biological Tissues: Statistical, Correlation and Topological Approaches" in Handbook of Coherent-Domain Optical Methods, pp. 107-148, Springer Science+Business Media New York (2013).

12. O. V. Angelsky, A. Ya. Bekshaev, P. P. Maksimyak, A. P. Maksimyak, S. G. Hanson, and C. Yu. Zenkova, "Self-diffraction of continuous laser radiation in a disperse medium with absorbing particles," Optics Express 21, 8922-8938 (2013).

13. Angelsky, O.V., Maksimyak, P.P., Perun, T.O., "Dimensionality in optical fields and signals," Appl Opt 32(30), 6066-71 (1993).

14. E. Wolf, "Unified theory of coherence and polarization of random electromagnetic beams," Phys. Lett. A. 312, 263-267 (2003).

15. J. Tervo, T. Setala, A. Friberg, "Degree of coherence for electromagnetic fields," Opt. Express 11, 1137-1143 (2003).

16. O.V. Angelsky, P.V.Polyanskii, C.V. Felde, "The emerging field of correlation optics," Optics and Photonics News 23, 25-29 (2012).

17. A. G. Ushenko, P. O. Angelsky, M. Sidor, Yu. F. Marchuk, D. R. Andreychuk, and N. V. Pashkovskaya, "Spatial-frequency selection of complex degree of coherence of laser images of blood plasma in diagnostics and differentiation of pathological states of human organism of various nosology," Appl. Opt. 53, B172-B180 (2014).

18. Yu. O. Ushenko, Yu. Ya. Tomka, I. Z. Misevitch, V. V. Istratiy and O. I. Telenga, "Complex degree of mutual anisotropy of biological liquid crystals nets", Opt. Eng. 50, 039001 (2011). 
19. Yu. A. Ushenko, Yu. Ya. Tomka and A. V. Dubolazov, “Complex degree of mutual anisotropy of extracellular matrix of biological tissues," Optics and Spectroscopy 110, 814-819 (2011).

20. A. Gerrard, J. M. Burch. Introduction to matrix methods in optics. New York.: A Wiley-Interscience Publication (1975).

21. J. W. Goodman. Statistical properties of laser speckle patters. In: Laser Speckle and Related Phenomena. Ed. J.C. Dainty, pp. 9-75, Berlin: SpringerVerlag (1975).

22. L. V. Kakturskiī, "Clinical morphology of acute coronary syndrome," Arkh Patol. 69, 16-19 (2007).

23. Cristina Basso, Fiorella Calabrese, Domenico Corrado, Gaetano Thiene, "Postmortem diagnosis in sudden cardiac death victims: macroscopic, microscopic and molecular findings," Cardiovascular Research 50, 290-300 (2001).

24. M.D. Pérez-Cárceles, J. Noguera, J.L. Jiménez, P. Martínez, A. Luna, E. Osuna, "Diagnostic efficacy of biochemical markers in diagnosis post-mortem of ischaemic heart disease," Forensic Science International 142, 1-7 (2004).

25. F. Martínez Díaz, M. Rodríguez-Morlensín, M.D. Pérez-Cárceles, J. Noguera, A. Luna and E. Osuna, "Biochemical analysis and immunohistochemical determination of cardiac troponin for the postmortem diagnosis of myocardial damage," Histol. Histopathol. 20, 475-481 (2005).

26. L. D. Cassidy, "Basic concepts of statistical analysis for surgical research," Journal of Surgical Research 128,199-206 (2005).

27. C. S. Davis, Statistical methods of the analysis of repeated measurements, New York: Springer-Verlag, 2002.

28. A. Petrie, B. Sabin, Medical Statistics at a Glance: Blackwell Publishing (2005). 